\title{
Ion Channelopathy Clinic-Sudden Death Genomics: An Impromptu Diagnosis
}

\author{
Mathew Schmidt ${ }^{1}$, Nachiket Patel $^{2}$ and Timothy E Paterick ${ }^{1 *}$ \\ ${ }^{1}$ Aurora Medical Center Green Bay, USA \\ ${ }^{2}$ Banner Medical Center Phoenix, USA
}

Submission: May 24, 2018; Published: August 06, 2018

*Corresponding author: Timothy E Paterick, Aurora Medical Center Green Bay, USA, Email: tpaterick@gmail.com

Abstract

Brugada syndrome (BrS) is an arrhythmogenic disorder often inherited in an autosomal-dominant fashion that is suspected when an Electrocardiogram (ECG) shows type 1 ECG pattern with ST-segment elevation in the right and anterior precordial leads. BrS is a clinical and ECG diagnosis. BrS is not a genetic diagnosis. Patients with BrS have an increased risk of syncope and sudden cardiac death owing to episodic ventricular tachyarrhythmia. The diagnosis of BrS is based on the presence of the type 1 Brugada ECG pattern, which may occur either spontaneously or after provocation tests with sodium channel blockers.

\section{Case Presentation}

\section{Clinical history}

24-year-old male had a salmonella infection presenting with fever and gastrointestinal symptoms of nausea, vomiting and diarrhea that persisted for 48 hours. He went to an urgent care center where during monitoring he had sudden loss of consciousness with ventricular fibrillation requiring cardiopulmonary resuscitation and 200 joules external defibrillation shock restoring sinus rhythm. Post shock it was noted his potassium was 3.2; magnesium 2.1 and troponin peaked at 53 approximately 30 minutes after the arrest. His echocardiogram revealed:

a. Normal LV systolic function and wall thickness. LVEF was $57 \%$.

b. Diastolic was functioning normal.

c. The right ventricle was thought to be enlarged with normal systolic function.

\section{d. Normal valve function.}

Cardiac cauterization revealed normal coronaries. The drug screen was negative. Patient was taking no medications. He denied smoking, alcohol use or illicit drug use. He had no risk factors for HIV. There was no family history for premature sudden death. Post arrest ECG revealed normal sinus rhythm, PR interval $198 \mathrm{msec}$, QRS $110 \mathrm{msec}$ with incomplete RBBB, and QT interval was $350 \mathrm{msec}$ with a corrected QT interval of $374 \mathrm{msec}$. Two days later the ECG showed sinus rhythm at 90 beats per minute with QRS 120msec with nonspecific IVCD pattern with QT interval was $380 \mathrm{msec}$. The $\mathrm{t}$ waves were tall with sharp ascending and descending limbs with symmetrical morphology. (Retrospective review suggests type 2 Brugada ECG) [1]. Prior medical history was significant for migraine headaches and acid reflux disease.

\section{Family history}

Maternal uncle with lone atrial fibrillation identified at age 33 years and required cardio version with subsequent bradycardia requiring permanent pacemaker. Maternal grandmother had a sister that passed away in infancy, reported due to SIDS.

\section{Physical examination}

The patient had a completely normal physical examination.

\section{Initial Assessment}

The initial QT interval (369-390msec) was borderline low during early acute post arrest period in the setting of fever and salmonella infection that normalized to $380-400 \mathrm{msec}$ range. There are three Brugada ECG patterns (Figure 1). The patient's initial ECG (retrospectively) revealed a type 2 Brugada pattern (Figure 2 \& 3). The patient had normal left heart structure and function and there was a question of RV enlargement by echocardiography. The latter raised a question of ARVD. Cardiac catherization revealed normal coronary arteries. The elevated troponin level raised a concern regarding coronary spasm in the setting of normal coronary arteries without narrowing or plaque. 


\section{Journal of Cardiology \& Cardiovascular Therapy}

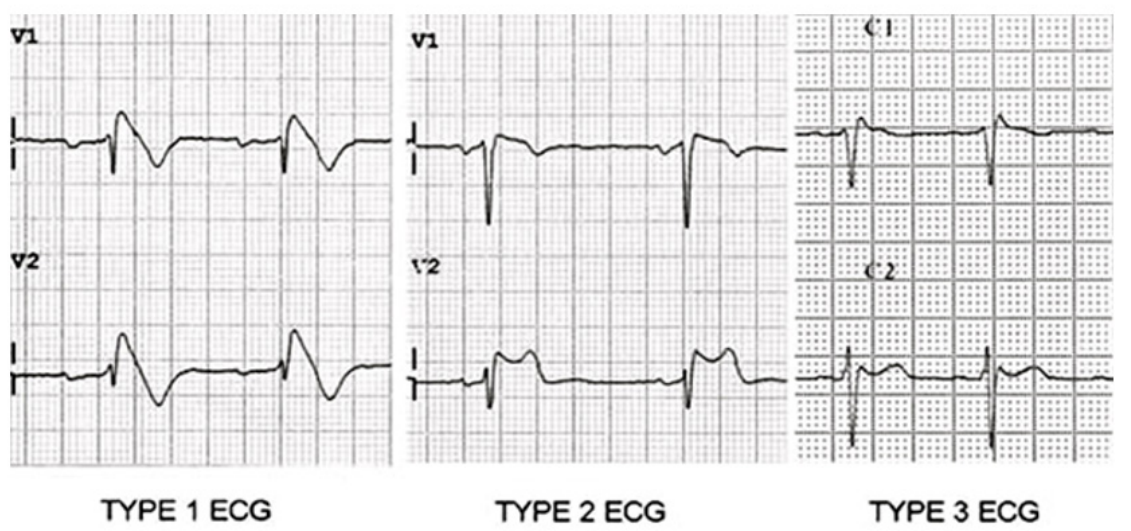

Figure 1: Classic type 1, type 2 and type 3 ECG brugada patterns.

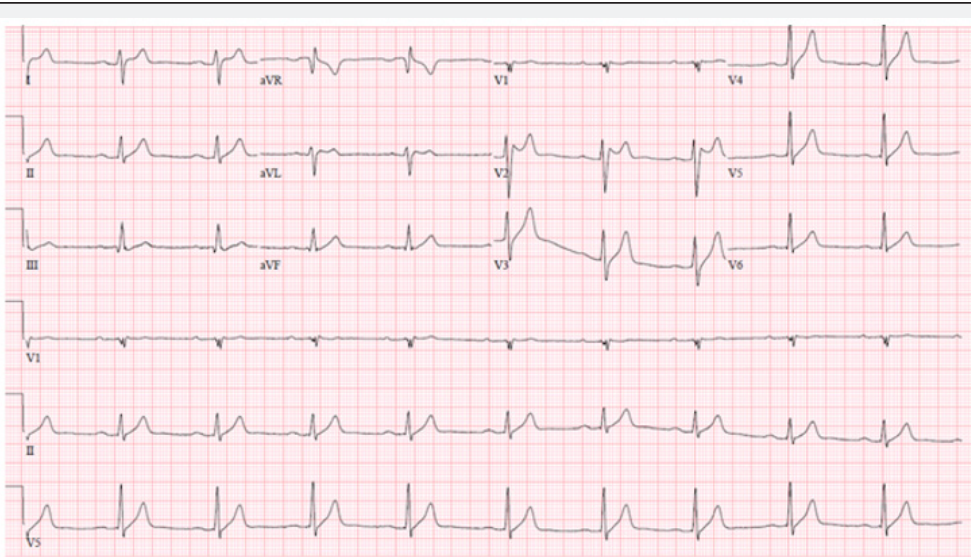

Figure 2: Initial ECG that has the type 2 ECG brugada Pattern.

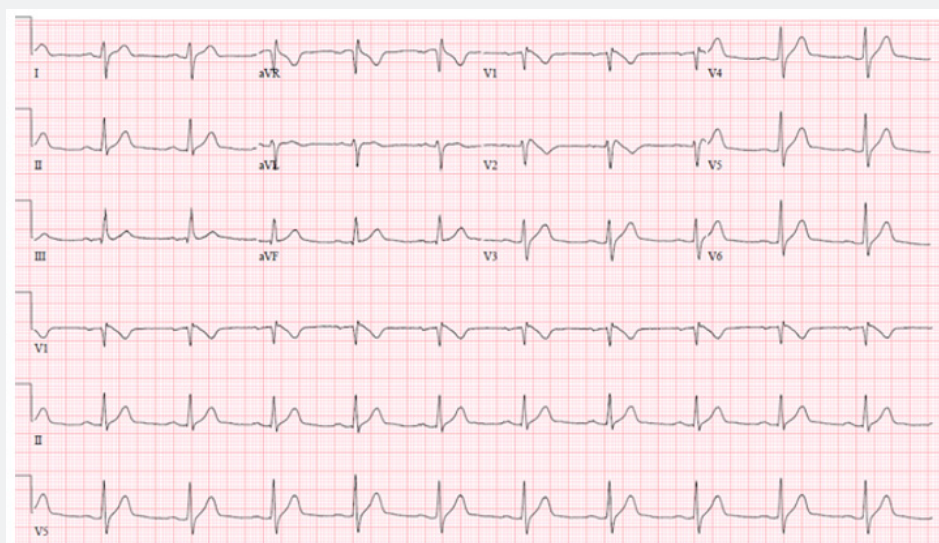

Figure 3: ECG after quinidine loading that unmasked the type 1 ECG brugada pattern.

\section{Genetic considerations when SQTS, ARVD and Brugada are being considered}

Genetic mutations for short QT syndrome:

a. Subunits of the potassium channel (gain of function) or calcium channel subunits (loss of function).

b. Calcium channel mutations with shorter than normal QT interval is associated with Brugada type J-point elevation in the right pre-cordial leads. c. RV enlargement made genetic assessment covering cardiomyopathies including ARVD cardio myopathy and LV non-compaction.

d. The putative genomic substrate for $\mathrm{Br}$ is: gene: SCN5A, locus: 3p21, protein: Na channel alpha subunit (NaV1.5). The diagnosis of BrS requires the type 1 ECG Pattern.

e. The family was informed that the short QT syndrome (SQTS) is very rare, with only 100 families diagnosed since 2000. There are three genes that have been found associated 
with SQTS, and the chances someone with true SQTS would have a positive genetic test is $15-20 \%$.

Plan

a) Combined Cardiac panel of 97 genes.

b) Parents and maternal uncle with lone atrial fibrillation identified at age 33 years have an ECG looking for Brugada pattern and QT analysis.

c) It was decided not to pursue provocation tests with sodium channel blockers.

d) Aggressive treatment of febrile illnesses with hydration and antibiotics.

e) Avoidance of drugs with cholinergic properties.

One month later after the salmonella infection was considered eradicated a Biotronik single chamber ICD was implanted.

2 month follow-up visit after genetic testing for ventricular fibrillation related cardiac sudden death

The Gene Dx 97 was performed, and no pathogenic variants were identified. Variants of uncertain significance in 3 genes were identified in desmin (DESc79G $>$ A $<$ p.Gly27Ser $>$ ), desmoplakin (DSPc4372C $>$ G $<$ p.Arg1458Gly), and sodium channel (SCN5A c5068-5070delGAC <p. Arg1458Gly>). These are variants of unknown significance (VUS) and are not known to be associated with pathogenic or benign outcomes.

\section{Overview}

The association of genes desmin, desmoplakin, and SCN5A with arrhythmogenesis is known, but the identified variants are not related to pathogenicity.

a. The desmin gene encodes for a muscle specific intermediate filament that is part of a network connecting myofibers to plasma membrane and mutations have been described with familial and cardiac myopathy. Review of the echocardiogram and his physical examination reveal preserved LV and RV function and no muscle weakness.

b. The desmoplakin gene mutation has been associated with arrhythmogenic cardiomyopathy and keratosis, such as palmoplantar keratosis and wooly hair. The patient and his family members had no skin or hair abnormalities and no echo features or rhythm disturbance to suggest ARVD cardiomyopathy.

c. SCN5A gene is associated with Brugada syndrome, atrial fibrillation, long QT and cardiomyopathy but it was felt there was no sodium channel-related disorder present on the ECG. (Retrospectively there was a type 2 Brugada pattern)[2].

The initial shorter QT interval during a febrile illness that normalized suggested this was not a fixed ion channel mutation. The potassium channel and calcium channel mutations that have been described with SSQTS were all negative.

\section{Further follow up}

One year after initial presentation the patient was admitted for quinidine loading by the electrophysiology team for recurrent shocks for polymorphic ventricular tachycardia. While being loaded with quinidine an ECG was obtained and it revealed the type 1 ECG pattern solidifying the diagnosis of BrS. Further investigation revealed his mother had a type 1 ECG pattern. The mother had no clinical events. Neither the patient's uncle nor father had a Brugada type 1 ECG [3].

\section{Discussion}

SCN5A targeted BrS genetic testing may be useful in patients in whom it has been established that there is a high index of suspicion for BrS based upon analysis of the patient's clinical history, family history, and expressed ECG or provocative drug testing ECG phenotype. Drug testing is not mandated in the setting of an isolated type 2 or type 3 Brugada ECG pattern. Mutationspecific genetic testing is recommended for family members and suitable relatives following the identification of a $\mathrm{BrS}$ causative mutation in an index case. Genetic testing is not recommended in the absence of an index case. BrS is characterized by right precordial ST elevation, associated conduction delays, potentially lethal arrhythmias, and a positive history for premature sudden death. BrS is typically expressed in males in the third to fourth decade of life. The prevalence of the disease is estimated to be 1 in 5,000 to 1 in 10,000 in Western countries. The prevalence of clinically silent type Brugada type 1 ECG pattern is likely much higher [4-6]. Risk stratification is based upon specific ECG patterns and symptoms and clinical considerations. Syncope and cardiac arrest put affected individuals at risk for lethal events $[5,6]$. Treatment of the high-risk subset of patient is ICD implantation. The yield of SCN5A genetic testing for strong clinical cases is $25 \%$. Thus, approximately $75 \%$ of $\mathrm{BrS}$ cases remain genetic in genetic purgatory [7].

The diagnosis of $\mathrm{BrS}$ is a clinical diagnosis and requires the type 1 Brugada ECG pattern in combination with unexplained syncope, family or personal history of premature sudden death. Genetic testing is not involved in the diagnosis. Genetic testing is most useful when one member of a family has been clinically diagnosed with Brugada Syndrome. If the mutation causing the disease in that person is identified, then the family members can be tested for the disease with genetic screening. One of the challenges of Brugada Syndrome is that ECGs findings are equivocal, or the pattern can come and go. A Type 2 or 3 Brugada Pattern, even in the setting of symptoms, is not sufficient to make the diagnosis. If a patient has a Type 2 or 3 Brugada Pattern, several maneuvers, such as moving the ECG leads higher in the chest can change the ECG to a classic Type 1 pattern. However, many times it is necessary to give these patients intravenous sodium channel blockers too turn the ECG into a more classic Type 1 pattern. The medications that are often used are flecainide or procainamide.

In our case treatment with an intravenous sodium channel blocker led to the fortuitous development of a type 1 Brugada ECG 
pattern solidifying the diagnosis of BrS. The patient fell into the $75 \%$ of BrS cases remaining in genetic purgatory. Often the key to diagnosis is careful ECG inspection. In our case the pursuit of genetic testing was debatable. Given the initial Type 2 Brugada ECG in a setting od sudden death it would have been prudent to obtain an ECG with the right precordial leads place one or two intercostal spaces higher and to consider intravenous sodium channel blocker in search of the Type 1 pattern to solidify the diagnosis of BrS. Once the diagnosis of BrS was made then it would be prudent to pursue genetic testing in the index case. If gene positive then family member should be encouraged to have gene testing.

\section{References}

1. Brugada P, Brugada J (1992) Right bundle branch block persistent ST segment elevation sudden cardiac death: a distinct clinical electrocardiographic syndrome. J Am Coll Cardiol 20(6): 1391-1396.

2. Antzelevitch C, Brugada P, Borggrefe M, Brugada J, Brugada R, et al. (2005) Brugada syndrome: report of the second consensus conference endorsed by the heart rhythm society and the European heart rhythm association. Circulation 111(5): 659-670.

3. Fowler SJ, Priori SG (2009) Clinical spectrum of patients with a Brugada ECG. Curr Opin Cardiol 24(1): 74-81.

4. Priori SG, Napolitans C, Gasparini M, Pappone C, Della BP, et al. (2002) Natural history of Brugada syndrome: Insights for risk stratification and management. Circulation 105(11): 1342-1347.

5. Gehi AK, Doung TD, Metz LD, Gomes JA, Mehta D (2006) Risk stratification of individuals with the Brugada electrocardiogram: A meta-analysis. J Cardiovasc Electrophysiol 17(6): 577-583.

6. Probst V, Veltmann C, Eckardt L, Meregalli PG, Gaita F, et al. (2010) Long term prognosis of patients diagnosed with Brugada syndrome: results from the Finger Brugada syndrome registry. Circulation 121(5): 635643.

7. Kapplinger JD, Tester DJ, Alders M, Benito B, Berthet M, et al. (2010) An international compendium of mutations in the SCN5A- encoded cardiac sodium channel patient s referred for Brugada syndrome genotype testing. Heart Rhythm 7(1): 33-46.

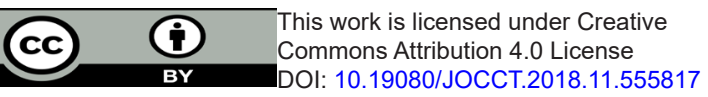

